\title{
NOTAS
}

\section{La idea de la justicia de Amartya Sen, un tratado sobre la injusticia}

\section{Rosa Colmenarejo Fernández'}

Resumen: En La idea de la justicia (2009), A. Sen retoma la idea de desigualdad como una forma de injusticia y señala las injusticias reparables, así como las fórmulas posibles para su análisis y compensación. La idea de la desigualdad es una constante en la obra de A. Sen desde Desigualdad económica (1972, revisada y ampliada en 1997). En Equality of what? (en castellano, Nuevo examen de la desigualdad) ampliaba aquella propuesta al analizar las implicaciones sociales de las desigualdades económicas, de los denominados "igualitarismos" y del "utilitarismo".

La medición de la desigualdad económica y de la pobreza permitió proponer un "corpus" conceptual al que pertenece el denominado "enfoque de las capacidades" (Capability approach), en el que colaboró activamente M. Nussbaum. Este "enfoque de las capacidades", aquello que somos capaces de ser, hacer y elegir, fue adoptado para el diseño de indicadores por el Programa de Naciones Unidas para el Desarrollo (PNUD) o el Índice Multidimensional de la Pobreza (S. Alkire y J. E. Foster, Oxford). La aplicación del enfoque de las capacidades permite identificar y ordenar alternativas de mejoras sociales adaptadas siempre al contexto en el que se generan las desigualdades.

Palabras clave: desigualdad, enfoque de capacidades, justicia.

Fecha de recepción: 16 de diciembre de 2012.

Fecha de admisión definitiva: I de junio de 2013.

1 Grupo de Estudios de Desarrollo GED. Facultad de Ciencias Económicas y Empresariales - Universidad Loyola Andalucía. Colaboradora honoraria del Área de Filosofía Moral de la Universidad de Córdoba 


\section{Amartya Sen's Idea of Justice, a treatise on inequality as a form of injustice}

Abstract: In The idea of justice (2009), A. Sen returns to the idea of inequality as a form of injustice, and highlights some repairable injustices as well as possible formulas for their analysis and compensation. The idea of inequality is a constant in the work of A. Sen since Economic Inequality (1972, revised and expanded in 1997). In Equality in what? (in Spanish, Nuevo examen de la desigualdad), he expanded on this proposal while analyzing the social implications of those economic inequalities denominated as "egalitarianism" and "utilitarianism".

This measuring of economic inequality and poverty led him to propose a conceptual "corpus" in which is found the so-called "Capability Approach", which he actively collaborated on with M. Nussbaum. This "capability approach" refers to what we are able to be, do and choose, and was adopted by the United Nations Program for Development (UNDP) to design the indicators in the Multidimensional Poverty Index (S. Alkire and JE Foster, Oxford). The application of the Capability Approach allows social improvement alternatives to be identified and managed, always adapting themselves to contexts that generate inequalities.

Key words: inequality, capability approach, fair and just.

\section{L'idée de justice d'Amartya Sen, un traité sur I'injustice comme inégalité}

Résumé: Dans L'idée de la justice (2009), A. Sen reprend l'idée de l'inégalité comme une forme d'injustice y les injustices repérables ainsi que les formules possibles pour leur analyse et leur compensation. L'idée d'inégalité est une constante dans l'œuvre de A. Sen depuis Inégalité économique (1972) révisé etapprofondie en 1997). Dans Equality of what? (en espagnol, Nouvel examen de l'inégalité), il approfondissait cette proposition en analysant les implications sociales des inégalités économiques, nommés "égalitarismes» et de «|'utilitarisme».

L'évaluation de l'inégalité économique et de la pauvreté a permis de proposer un "corpus» conceptuel auquel appartient ce qui est appelé "approximations des capacités», et pour lequel $M$. Nussbaum a collaboré activement. Cette "approximation des capacités», c'est-à-dire ce que nous sommes capables $d^{\prime}$ être, faire ou choisir a été adopté pour la conception d'indicateurs pour le Programme des Nations Unies pour le Développement (PNUD) ou I'Indice Multidimensionnel de la Pauvreté (S. Alkire et J- E. Foster, Oxford . L'application de l'approximation des capacités permet d'identifier et d'ordonner des alternatives $d$ 'améliorations sociales, toujours adaptées au contexte où se créent les inégalités

Mots clefs: Inégalité, approximations des capacités, justice. 


\section{Introducción}

En un número especial de la revista Human Development 9 (2008), n 3, editado en ocasión de la conferencia, correspondiente al año 2007, de la Asociación Internacional de Desarrollo Humano y Capacidades (HDCA), fue publicado el artículo firmado por Amartya Sen, catedrático de Economía y Filosofía en la universidad de Harvard y premio Nobel de Economía en 1998, con el título "The Idea of Justi$\mathrm{ce}^{\prime \prime}$, en él se avanzaba gran parte de los temas que abordaría un año después en forma de ensayo. La idea de la justicia ${ }^{2}$ es en gran parte un libro cuyo contenido procede de artículos publicados previamente en revistas especializadas, algunos de ellos hace más de diez años, pero ello no merma un ápice el interés que tiene como obra completa. En primer lugar, porque el autor ha realizado con éxito la ingente labor de ordenar convenientemente contenidos de diversa procedencia, de tal suerte que la obra se lee como el autor pretende, como un tratado de justicia. Además, ofrece la posibilidad de abordar en castellano una gran obra que de otro modo, además de dispersa, solo encontraríamos en inglés ${ }^{3}$. Finalmente, y siguiendo con la tradición anglosajona de discusión, crítica y respuesta públicas, nos ofrece un texto que ha pasado por avatares como una conferencia mundial sobre desarrollo, una publicación previa y una crítica meticulosa por colegas y compañeros, de todos y cada uno de los artículos que la comprenden, según reconoce el autor en los agradecimientos. La idea de la justicia ha sido construida en el tiempo y en el espacio, y quizá en ello resida gran parte de su encanto coral. Leyéndola no nos parece escuchar solo a Sen, sino a muchos otros pensadores, occidentales y orientales, actuales y de la antigüedad.

En la primera página del prefacio, el autor establece ya las dos premisas fundamentales para la nueva teoría que pretende su idea de la justicia. Primero, que es la identificación de la injusticia reparable lo que nos mueve a pensar en la justicia. Segundo, que hay una necesidad de razonamiento e imparcialidad, en tanto que examen crítico, no solo para comprender los conceptos éticos de la injusticia, sino también para explicar la emotividad que generan. Solo mediante la argumentación razonada, con nosotros mismos y con los otros, afirma Sen, podremos considerar posiciones enfrentadas y superar la extendida "tolerancia indiferente". Las partes primera y segunda, que comprenden diez capítulos, abordan estas dos cuestiones

\footnotetext{
${ }^{2}$ A. SEN (2010) La idea de la justicia, Madrid, Taurus, 499 pp., traducción del inglés de Hernando Valencia Villa

3 "Seniana" es una página web muy activa que aloja estudios y recursos sobre Amartya Sen en español: http://www.uco.es/amartya_sen/
} 
fundamentales. Primero en "Las exigencias de la justicia", y después en "Formas de razonamiento". Consideramos aquí entonces que la obra que nos ocupa es un tratado sobre la injusticia, pues Sen aboga por la justicia basada en realizaciones y no por aquella justicia "ideal" o trascendental basada en las instituciones que representa la obra de autores precedentes, con especial atención a John Rawls.

Dado que el pensamiento de Amartya Sen se centra en las personas, se considera aquí que muchas de estas injusticias, más que con insuficiencias institucionales, guardan estrecha relación con la forma en que las personas viven sus vidas. La idea de la justicia pone el énfasis en las vidas que la gente es capaz de vivir, cuyos logros y limitaciones se estudian en los capítulos 11 a 14, y que conforman la tercera parte del libro, denominada "Los materiales de la justicia".

Finalmente, en "Razón pública y democracia", capítulos 15 a 17, se discute la funcionalidad de las instituciones en esta idea de la justicia, donde la democracia debe proveer suficientes espacios de discusión pública más allá de su "institucionalidad". Sen defiende, además que esta forma de gobierno por discusión, una idea tomada de Walter Bagehot, permitiría impulsar la democracia global y con ella, la justicia global.

Como ya hemos comentado aquí, Sen pretende en La idea de la justicia ${ }^{4}$ incluir fórmulas para superar la injusticia, más que definir la justicia perfecta en sociedades bien ordenadas, en clara alusión al enfoque trascendental que adopta John Rawls en La teoría de la justicia (1971). El libro está dedicado, sin embargo, a su memoria, y es con él con quien establece una conversación crítica continua en torno a los asuntos fundamentales abordados en una teoría que no llegaba a considerarse

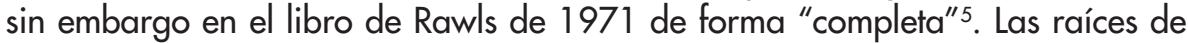
este renovado enfoque contractualista, que representa John Rawls, las ubica Sen en Thomas Hobbes, John Locke, Jean Jacques Rousseau e Immanuel Kant.

Sen reconoce la influencia de éstos en su propia obra, pero adoptando sin embargo, lo que él denomina la tradición ilustrada alternativa, o comparatista,

${ }^{4} \mathrm{~A}$ partir de aquí las referencias a citas textuales, que corresponden a la edición en español de 2010 , se indicarán dentro del texto únicamente como número de página entre paréntesis. En aquellos casos en los que se considera necesario hacer referencia a la obra original se indicará a continuación. La edición original que se sigue es A. SEN (2009) The Idea of Justice, Londres, Penguin.

${ }^{5}$ Consideramos que completan la mencionada Teoría de la justicia de John RawLs sus escritos Liberalismo político (1993), El derecho de gentes. La idea de la razón pública revisada (1999) y Justicia como equidad (2001), concebidos en respuesta a las críticas recibidas a su primera obra, en lo que el propio Rawls denominaría "extensiones" de su teoría. 
basada en realizaciones, donde ubica enfoques tan variados como los aportados por Adam Smith, Marie Jean Antoine Nicolas de Caritat, Marqués de Condorcet, Mary Wollstonecraft, Jeremy Bentham, Karl Marx o John Stuart Mill, autores que, como él mismo, propusieron distintas formas de comparación social basándose en las injusticias observadas. Sen los agrupa siguiendo este criterio, aun dejando documentada constancia de las notables distancias que existen entre sus formas de comprender la justicia.

Simultáneamente, fluyen en La idea de la justicia ideas procedentes de la historia intelectual de la India y de otras fuentes no occidentales que hacen florecer la comprensión y la empatía: Gautama Buda, Akbar, Ashoka, Kautilya de la India, Alejandro, Aristóteles o Kenzaburo Oé. Su presencia refuerza e ilustra la tesis de Sen, esto es, que en muchas y diferentes partes del mundo se han explorado ideas similares o cercanas sobre justicia, equidad, responsabilidad, deber, bondad y rectitud. En ello basará la universalidad de su enfoque centrado en las capacidades de las personas, con la agencia y la libertad como principios esenciales.

Sen invoca el papel central de la razón en la comprensión de la justicia, la esperanza en un mundo menos injusto reside en la discusión pública razonada. Por ello, rechaza la creencia de que las cuestiones sobre valores tengan una sola respuesta correcta. Su investigación trata sobre cómo llegar a acuerdos razonados para reducir la injusticia, manteniendo además que estos acuerdos pueden ser alcanzados a pesar de las diferencias posibles entre los interlocutores sobre un ideal de justicia. El problema planteado en la introducción, sobre tres niños y la flauta que se disputan, ilustra perfectamente la posición que defiende Sen, un acuerdo imparcial no puede surgir de un esquema social perfectamente justo e identificable (44-47).

\section{Contenido de La idea de la justicia}

El capítulo 1, "Razón y Objetividad", cuestiona la crítica a la que se ha visto sometido el razonamiento racional ilustrado. Se trata de un trabajo que fue publicado en el año 2000 en New York Review of Books, como reseña bibliográfica a la obra de Jonathan Glover, The Reach of Reason: East and West, y reeditado como capítulo del ensayo La argumentación india ${ }^{6}$. Akbar, emperador mogol de la India en el

${ }^{6}$ A. SEN (2005) La argumentación india, Barcelona 2007, Gedisa. En aquellas obras en las que existe edición en español, se indica primero el año de su publicación en el idioma original y al final el año de su traducción. 
siglo XVI, para quien la senda de la razón, o la regla del intelecto, han de ser el factor clave del comportamiento bueno y justo, es aludido por Sen en su argumento a favor de que en el escrutinio razonado no radica ninguna forma segura de hacer de modo correcto las cosas, sino en ser tan objetivos como razonablemente podamos (70). Los distintos enfoques de objetividad abordados, Akbar, Rawls, Putnam, Smith y Sen mismo, coinciden en la necesidad de un encuentro razonado sobre una base imparcial, cuyo requerimiento ha de satisfacer el razonamiento empleado en pos de una objetividad ética. En esa búsqueda de objetividad ética, afirma Sen siguiendo a Hilary Putnam ${ }^{7}$, aun asumiendo su inaccesibilidad, es preciso dotar de transparencia las cuestiones planteadas sometiéndolas a un escrutinio adecuado.

La idea básica que se aborda en el capítulo 2, denominado "Rawls y más allá" es la de equidad, en la que se basa no solo la teoría de la justicia de Rawls, sino también la que nos presenta Amartya Sen. La idea de equidad precede a la de justicia a través de la imparcialidad, o ausencia de intereses creados en la toma de decisiones. Para alcanzarla diseñó Rawls el experimento denominado "el velo de la ignorancia", o "posición original", que consiste en un estado imaginario de ignorancia, esencialmente respecto a los intereses personales y opiniones respecto a la buena vida, que permitiría adoptar un solo conjunto de principios de justicia por unanimidad. Sen se muestra especialmente crítico con este tipo de imparcialidad, a la que denomina "cerrada", en los capítulos 5 y 6 de esta obra, aunque la primera crítica a la posición rawlsiana se remonte a 1965 y haya sido el tema principal en al menos otro artículo de Sen y le haya dedicado otro capítulo completo de su Elección colectiva y bienestar social ${ }^{\beta}$.

"Instituciones y personas" es el título del capítulo 3, que comienza con un análisis de dos perspectivas sobre la justicia en la India, que se remontan al siglo II a.C.; por un lado la propuesta de Ashoka, quien creía firmemente que era posible promover la justicia social desde la voluntad ciudadana de buen comportamiento, frente a la economía política de Kautilya, que veía en las instituciones y en la regulación mediante incentivos y castigos, el único modo de alcanzar una sociedad justa. Sen nos muestra como estos dos papeles que juegan instituciones y personas, no solo están firmemente interrelacionadas, sino que mantienen su plena vigencia en señalar la filosofía política actual. El capítulo se centra en explicar esa interrelación y en el espacio de encuentro, lejos de considerarlas propuestas enfrentadas. Lo

${ }^{7}$ H. Putnam (2004) Ethics Without Ontology, Cambridge (EE.UU.), Harvard University, p.75.

${ }^{8}$ A. SEN (1970) Elección colectiva y bienestar social, Madrid 1976, Alianza. 
que subyace aquí, apunta Sen, es la relación entre el comportamiento real y la elección de instituciones idealmente justas. Así, mientras Rawls mantiene que, una vez conformadas, las instituciones justas permitirán, en una sociedad razonable y perfectamente democrática, que los ciudadanos se olviden de sus intereses personales tanto como confíen en la relación contractual que han acordado con las instituciones que ellos mismos han contribuido a establecer, Sen mantiene que la distancia entre este mundo idealizado y trascendental que Rawls construye para su idea de la justicia es demasiado elevada como para incidir en la justicia social basada en realizaciones, pues es en la idea de la injusticia en la que Sen pretende apoyarse como modo de promover la justicia. Sen es concluyente respecto a la necesidad de ir más allá de una justicia confinada en las instituciones, por muy correctas que éstas sean, y no dejar de cuestionar nunca cómo van realmente las cosas y si es posible mejorarlas.

En "Voz y elección social", capítulo 4, Sen argumenta sobre la dependencia que una teoría de la justicia tiene tanto de una exposición razonada como de un diálogo abierto a la discusión y a la crítica. La teoría que defiende Sen pretende ser lo suficientemente amplia como para comprenderse como inacabada. En esto no difiere ciertamente de la de Rawls, quien se esforzó en rebatir, y en la mayor parte de los casos, admitir, todas y cada una de las extensiones a su teoría que se le fueron sugiriendo. Así, mientras que en el contractualismo, que tiene en Rawls su mejor exponente contemporáneo, la búsqueda se orienta hacia la identificación de la naturaleza de lo "justo", el enfoque de la justicia que propone Sen, sigue una ruta comparativa en lugar de trascendental, centrándose en las realizaciones reales de las sociedades más que en sus instituciones y sus reglas, busca criterios para considerar una opción "menos injusta" que otra. El capítulo está centrado en mostrar las aportaciones que la teoría de la elección social desarrollada en el siglo XVIII por Condorcet y Jean-Charles de Borda, y retomada por Kenneth Arrow en 1950, realiza para indagar un marco para la razón pública. La disciplina formal de la elección social consiste en la posibilidad de agregar juicios individuales en grupos de diferentes personas, mediante el lenguaje matemático, con la intención de obtener un modelo, o construcción razonada, del orden social.

El capítulo 5, "Imparcialidad y objetividad", se inicia resaltando como la tradición política y moral contemporánea ha seguido la orientación de Mary Wollstonecraft, al considerar a toda persona como política y moralmente relevante. Esta "universalidad" de la dignidad humana, es parte integral de la imparcialidad que defiende Amartya Sen.

Hay dos cuestiones de "no subjetividad" posibles, que Sen encuentra aquí: la primera se refiere a la comprensión y comunicación interpersonal de un lengua- 
je de justicia e injusticia, que no se vea confinado a una subjetividad personal impenetrable, sino todo lo contrario. La segunda es una aceptabilidad objetiva, que implica que las personas pueden participar en debates sobre la corrección de las tesis formuladas por diferentes personas. Tanto la tesis de Wollstonecraft sobre la corrección esencial de incluir a todas las personas en los planteamientos de la moral y la política, o la afirmación de Sidgwick sobre la verdad de la universalidad y la imparcialidad, implican cuestiones de comprensión interpersonal y veracidad general, o "aceptabilidad objetiva". Sen considera que aunque estas dos formas están interrelacionadas, no son exactamente iguales e intenta aclarar esta distinción en los dos siguientes epígrafes. En primer lugar, esa comprensión es posible en tanto se pertenece a un grupo social con un lenguaje y una concepción del mundo determinados. Esto es, nuestras valoraciones éticas están mediatizadas por la comprensión de las convenciones y el lenguaje que empleamos para comunicarnos. Para ilustrarlo emplea Sen dos obras de Wittgenstein: mientras el Tractatus trata de ver el lenguaje aislado de las circunstancias sociales en las cuales se emplea, las Investigaciones filosóficas enfatizan las convenciones y reglas que dan a las expresiones su significado particular. Vivian Walsh se refiere a estas interrelaciones como un "embrollo" al que estamos sometidos de ordinario entre "hecho, convención y valor"

La aceptabilidad objetiva de una determinada propuesta ética pasa en primer lugar, como hemos visto, por su comprensión a través de una comunicación adecuada. Hay una prueba adicional, sugiere Sen, que trata de la aceptabilidad pública, con la habilidad para enfrentarse al razonamiento público abierto, y de exponer suficientes (razones) para convencer a todas las personas razonables de que es razonable ${ }^{10}$.

Sen considera dos formas de imparcialidad: "cerrada" y "abierta". La primera está fundamentada en lo que Rawls denominó "posición original", un procedimiento

9 V. WALSH (2003) "Sen after Putnam": Review of Political Economy, 15, $\mathrm{n}^{\circ}$ 3. La autora trata aquí dos formas de enfrentarse a los valores morales en economía. De un lado, Amartya Sen quien, apoyado en los planteamientos neoaristotelistas de Martha Nussbaum, ha actualizado los valores morales de la economía clásica que residen en Adam Smith. El otro protagonista es Hilary Putnam, quien en su libro The collapse of the fact/value dichotomy, rebate el argumento que considera que únicamente "un hecho es cierto, mientras que un valor es un valor y nunca llegarán a encontrarse".

$10 \mathrm{~J}$. Rawls (1993) El liberalismo político, Crítica, Barcelona, 2004, 151. Sen considera que Rawls se refiere a personas razonables como "aquellas que estén dispuestas a comprometerse con la discusión pública, examinar los argumentos y las pruebas disponibles, y razonar sobre ellos con la mente abierta"; aunque se muestre comprensivo aquí, empleará este argumento más adelante para criticar "la posición original". 
mental para hacer juicios imparciales que invoca únicamente a los miembros de una sociedad o nación determinada, "un pueblo dado", destinatario final del juicio emitido, expuesto en su Teoría de la Justicia (1971). Por otro lado, está la imparcialidad "abierta", donde las evaluaciones imparciales precisan invocar juicios externos al grupo local para evitar el "prejuicio parroquial"11. Adam Smith propuso la figura del "espectador imparcial" en su Teoría de los sentimientos morales"12, un espectador "justo", que puede proceder de lejos o de la propia comunidad, nación o cultura, capaz de enunciar juicios imparciales.

De la comparación entre ambos experimentos mentales se ocupa el capítulo 6: "Imparcialidad cerrada y abierta". El experimento mental que propone Smith, requiere "examinar nuestra propia conducta como imaginamos la examinaría cualquier otro espectador justo e imparcial". Sen sugiere, como ya hizo Rodríguez Braun en la introducción a la edición española de la obra de Smith, que el enfoque del imperativo categórico kantiano, esto es "Obra sólo según una máxima tal que puedas querer al mismo tiempo que se torne ley universal", tiene una clara influencia smithiana, y que muy probablemente en ella se encuentre la semilla de la insistente búsqueda de la imparcialidad en la filosofía política actual.

La segunda parte, denominada "Formas de razonamiento", incluye cuatro capítulos. El primero de ellos, corresponde al capítulo 7, se denomina "Posición, relevancia e ilusión" y en él muestra Sen la importancia de la empatía para la idea de la justicia que defiende. El autor trata de comprender la "otredad" como factor relevante en la toma de decisiones justas, es decir, respecto a la imparcialidad abordada en el capítulo precedente. El término que emplea para referirse a las posiciones adoptadas desde una perspectiva de poder es el de "ilusión", que toma de la teoría marxista. Una "ilusión objetiva", o ilusión de objetividad, es una creencia posicionalmente objetiva cuando el diagnóstico transposicional de dicha creencia es, en efecto, equivocada (193). Aborda Sen, entonces asuntos como la salud, la morbilidad o la discriminación de las mujeres. El capítulo se cierra con un recorrido sobre la idea del "vecino" en la historia del pensamiento moral, centrándose muy especialmente en pasajes del Nuevo Testamento.

${ }^{11}$ El término parochialism, que emplean tanto Smith como Sen, podría ser traducido como "provincialismo" o "localismo". Empleamos aquí, sin embargo, "parroquialismo", tal y como figura en las ediciones en castellano de las obras de estos autores.

${ }^{12}$ SMITH, A. (1759) La teoría de los sentimientos morales, edición a cargo de Carlos Rodríguez Braun, Alianza, Madrid, 1997.

Revista de Fomento Social 68 (2013) 
El capítulo 8, "La racionalidad y las otras personas", trata la conceptualización crítica de la racionalidad en la historia del pensamiento. Ello le permite considerar la distinción entre "elección racional" y "teoría de la elección racional". Ésta última caracteriza la racionalidad de la elección simplemente como una maximización del propio interés, y aquí detecta Sen una devaluación importante del razonamiento humano. Frente a ésta teoría, claramente utilitarista, desarrolló Sen, en 1977, una conferencia con el explícito título "Tontos racionales" ${ }^{13}$, en el que realizó una distinción entre la idea smithiana de simpatía y la de compromiso. El mismo tema fue abordado en Rationality and Freedom ${ }^{14}$, donde Sen considera que no hay nada irracional en hacer elecciones que excedan los estrechos límites de la búsqueda exclusiva del interés propio.

Las conclusiones sirven aquí de pie al siguiente capítulo, el número 9, "La pluralidad de las razones imparciales". El problema de la posición y la imparcialidad es planteado de nuevo a través de un ejemplo literario tomado de Shakespeare, La vida y la muerte del rey Juan: "Mientras sea un mendigo, despotricaré y diré que no hay otro pecado sino el de ser rico; y cuando sea rico, mi virtud consistirá en decir que el único vicio es la pobreza". El problema así planteado se adapta a la propuesta de Rawls del velo de la ignorancia, ideado precisamente para que la gente pueda ver más allá de sus intereses personales. Sen considera, sin embargo, realizando un mínimo tratamiento al asunto de la cooperación, que hay muchas situaciones cotidianas en las que no es el interés lo que mueve a las personas a actuar, sino un afán de solidaridad espontánea, una acción colectiva que procede de normas sociales de comportamiento no escritas, como ha estudiado Elinor Ostrom ${ }^{15}$. El beneficio mutuo, como defiende el contractualismo, ya sea hobbesiano o rawlsiano, no es el único que ofrece un fundamento racional al comportamiento no interesado.

"Realizaciones, consecuencias y acción", es el título del capítulo 10. A partir de un poema épico indio, el autor nos plantea dos puntos de vista, dos posiciones morales, que se corresponden con una deontología independiente de las conse-

${ }^{13}$ SEN, A. (1977) "Rational Fools: A Critique of the Behavioural Foundations of Economic Theory", Philosophy and Public Affairs, 6.

${ }^{14}$ A. SEN (2002) Rationality and Freedom, Cambridge, Harvard University.

${ }^{15}$ Premio Nobel en Economía en 2009, por su "análisis de la gobernanza económica, especialmente de los recursos comunes (the commons). Autora, entre otros, de Governing the commons: The evolution of institutions for Collective action. [Political Economy of Institutions and Decisions series], Cambridge (1990), Cambridge University. 
cuencias frente a una evaluación sensible de las consecuencias de la acción, o razonamiento consecuencialista. Sen desgrana el alcance de los argumentos de ambos guerreros, apelando al lector con dilemas y planteamientos que permiten una ilusión de diálogo. Este capítulo es quizá el más didáctico, pues se exponen de un modo bastante claro los problemas y se dirimen con argumentos fácilmente comprensibles.

La tercera parte, denominada "Los materiales de la justicia", comprende cuatro capítulos. En el capítulo 11, "Vidas, libertades y capacidades", se exponen los fundamentos del enfoque de las capacidades humanas. Frente a indicadores económicos como el Producto Interior Bruto, el enfoque de las capacidades pretende un reconocimiento de indicadores directos que permitan medir la calidad de vida, el bienestar y las libertades que las vidas humanas pueden traer consigo. La libertad de las personas es para el enfoque de las capacidades un agente de cambio, una oportunidad para llevar una vida que valoremos. Se transforma así en el foco de información, y con ello las capacidades humanas quedan vinculadas estrechamente a la oportunidad de libertad, como oportunidad comprehensiva, es decir, más allá del objetivo último. Esta característica hace que las capacidades sean atributos personales y no colectivos, pero no se trata sin embargo de una propuesta individualista. Sen mantiene que la persona debe estar en el centro, si bien es preciso reconocer la profunda interdependencia de las valoraciones entre individuos.

Amartya Sen considera que es la libertad para valorar las oportunidades particulares que más nos convienen, y que no tienen por qué estar directamente relacionadas con su contribución a nuestro nivel de vida, lo que nos permite desarrollar un sentido de la responsabilidad como el que precisa la conservación del medioambiente, la observación de los costes ambientales y sociales en los balances económicos o el desarrollo sostenible, entre otros. En este sentido y en una perspectiva en deuda con Rousseau y Kant, Sen cita a Luc Ferry quien argumenta que lo que realmente distingue al ser humano como ser moral, no es la razón ni el lenguaje sino, precisamente, la libertad o "buena voluntad", es decir, la capacidad para actuar de forma desinteresada en nuestra relación con la naturaleza ${ }^{16}$.

En el capítulo 12, "Capacidades y recursos" aborda Sen un tema al que ha dedicado gran parte de sus trabajos: la pobreza y las hambrunas. Sen considera la pobreza como una privación de capacidad, y no tanto como una limitación en

${ }^{16}$ FERRY, L. (1992) El nuevo orden ecológico. El árbol, el animal y el hombre, Barcelona 1994, Tusquets, pp. 74 y 77.

Revista de Fomento Social 68 (2013) 
el ingreso, con una gran incidencia por contingencias ambientales y sociales. En primer lugar, la heterogeneidad personal, pero también las condiciones ambientales, tanto físicas, como climáticas, como de calidad y salubridad del entorno, entre ellas la contaminación o la limitación en el acceso a los recursos naturales. También la estabilidad del clima social, en especial en lo referente a la asistencia sanitaria y recursos en educación. Finalmente, lo que Sen denomina "diferencias en las perspectivas relacionales", y que se refiere al coste en arreglo personal, unos recursos siquiera mínimos, que se precisa para estar integrado socialmente. El capítulo prosigue con una mínima referencia al problema de la discapacidad como limitación de capacidad, y una revisión crítica sobre cómo han abordado autores como Rawls y Dworkin, el acceso a los bienes primarios. La propuesta de Sen consiste en incorporar el enfoque de las capacidades a ambos problemas, sin especificar claramente su propuesta para llevarlo a cabo.

"Felicidad, bienestar y capacidades", es el capítulo 13. La felicidad ha sido el objeto de la corriente filosófica, el utilitarismo, con la que Sen se ha mostrado más crítico. En este capítulo desgrana los argumentos de su crítica, desde la conceptualización y alcance de la felicidad, sobre su valoración y sobre la economía del bienestar que provocó la asunción de la filosofía utilitaria. Así presenta las capacidades humanas, que defienden su propio enfoque, frente a las "ventajas" que suponen la felicidad y el bienestar. Finalmente aborda la relación del bienestar con la libertad, para concluir, que los objetivos y las prioridades humanas se dirigen muchas veces mucho más allá de los estrechos límites del bienestar personal (320).

"Igualdad ("equity" en el original) y libertad" son los asuntos abordados en el capítulo 14. La relación entre el enfoque de las capacidades y la igualdad, basado en un singular trabajo previo del autor denominado "Iguales a qué" 17 , concluye que la exigencia de considerar a las personas como iguales se refiere a la exigencia normativa de imparcialidad, así como a las reivindicaciones asociadas de objetividad (323). El enfoque de las capacidades no es un enfoque igualitario, dado que en la idea de capacidad tiene un gran peso el aspecto de oportunidad de la libertad, lo que nos permite evaluar las ventajas y desventajas de la persona. Sen comprende la igualdad como un espacio multidimensional, por ello su crítica está dirigida hacia la visión unifocal, que supone su generalización, de la igualdad como enfoque. Las ideas de Sen respecto a la libertad y la igualdad, es que deben ser, al igual que la idea de la justicia en la que necesariamente han de estar

17 A. SEN (1980) "Equality of What?, en S. MCMURRIN ed. (1980) Tanner Lectures in Human Values, Cambridge, Cambridge University (EE.UU.), vol. 1. 
insertas, multidimensionales y suficientemente espaciosas como para permitir un razonamiento público suficiente, que garantice con ello la democracia. De esta relación se ocupa la cuarta parte del libro: "Razón pública y democracia".

El capítulo 15, "La democracia como razón pública", aborda el problema de la democracia como un lugar de discusión pública, más allá de un recuento formal de votos. La democracia, entonces, en conexión con la idea de justicia. Para argumentar esto nos remite al pensamiento de autores contemporáneos como Rawls, Habermas, Ackerman, Sheyla Benabib, Cohen o Dworkin. El siguiente paso es ubicar los orígenes globales de la democracia, pues aún reconociendo el valor de Grecia, Sen ve necesario introducir en la historia, la tradición democrática municipal india, o el sistema de elecciones practicado en otras sociedades no occidentales. Sen no considera la democracia, pues, como un puro fenómeno regional, y se emplea a fondo en documentarlo. El capítulo concluye con un epígrafe dedicado a la contribución de los medios de comunicación para el éxito de este "gobierno por discusión" que supone la democracia real.

El capítulo 16, "La práctica de la democracia", comienza con un relato sobre la experiencia que tuvo Amartya Sen en su infancia, la conocida como "hambruna de Bengala" que tuvo lugar en 1943. Sen achaca la gravedad alcanzada por la hambruna a la incapacidad del gobierno británico primero para anticiparse, al no tomar decisiones sobre cuestiones que afectaban a bienes básicos como el arroz, $y$ después para reconocerla a tiempo y tomar las medidas oportunas. Miles de personas morían en las calles cada día, mientras los técnicos y miembros del gobierno planificaban la redistribución de alimentos sin tener en cuenta lo que la población estaba sufriendo. En el capítulo anterior Sen expuso la idea de que, efectivamente, nunca se dio una hambruna en un país con una democracia "funcional", con elecciones periódicas, partidos de oposición, libertad de expresión y medios de comunicación relativamente libres. El sistema democrático está por tanto íntimamente ligado al concepto de desarrollo, y con él al de seguridad humana y control sobre el poder político. El epígrafe final está dedicado al asunto más espinoso al que han de enfrentarse las democracias modernas, incluyentes por mandato constitucional, que no es otro que el de las minorías y sus prioridades. Unas prioridades que, como ya avanzó Condorcet en el siglo XVIII, no pueden ser legítimamente sacrificadas por las prioridades de los muchos (384). Sen no confía en las instituciones para hacer efectiva la justicia, sino en el reconocimiento de (nuestros) patrones reales de conducta y del funcionamiento de las interacciones políticas y sociales. La justa actuación de una institución depende de las personas y de sus capacidades. Sen concluye invocando a los nyaya, justicia basada en realizaciones, y no solo a los niti, justicia basada en ideales, que nos fueron pre- 
sentados en la introducción, para así fundamentar una idea de la justicia a través de la observación de las injusticias.

El capítulo 17, "Derechos humanos e imperativos globales", está dedicado a las sucesivas declaraciones sobre derechos humanos que han tenido lugar en la historia occidental, y que son presentadas como invitaciones a una legislación nueva. Aún reconociendo el valor que estas declaraciones han tenido para la revisión efectiva de determinados entramados legislativos, Sen se muestra crítico y propone superar esa ruta legislativa, que se entiende como un fin en sí misma, a través del enfoque de las capacidades humanas. Sen considera que sería mucho más efectivo alcanzar ese tipo de acuerdos y consensos desde la denuncia, la crítica en los medios, la agitación y, muy especialmente, a través de los debates públicos. Siguiendo los argumentos expuestos a lo largo de este ensayo, concluye que los derechos humanos solo encontrarán un acomodo ético en tanto en cuanto sean sometidos a un escrutinio público razonable.

El capítulo 18, "La justicia y el mundo", lo emplea el autor para realizar una retrospectiva de todos los temas abordados en el ensayo. Comenzando por la conceptualización de injusticia, el alcance del razonamiento, la visibilidad necesaria de la justicia, la defensa de la pluralidad de razones, que no debe ser confundida con la "tolerancia", las ideas de imparcialidad abierta y cerrada y el parroquialismo, así como los marcos comparativos, la idea de la democracia y su conexión con la idea de la justicia, la idea del razonamiento global y, finalmente, la idea de contrato social frente a la de elección social.

\section{Conclusiones}

Amartya Sen propone un enfoque en el que la justicia no es teorizada como un ideal a alcanzar sino como un complejo procedimiento para reconocer aquello que es injusto. Para ello, expone el modo en que puede quedar definida la injusticia mediante herramientas como la argumentación pública razonada, la idea de imparcialidad y su estrecha relación con la idea de objetividad. El único sistema que facilita esta tarea es la democracia, solo en el seno de una democracia mínimamente decente es posible alcanzar un consenso sobre cuáles son aquellas injusticias reparables. El esfuerzo por evitar que su teoría sea una teoría política normativa, pues su propuesta pretende sentar las bases para una teoría comparativa, no impide sin embargo que a lo largo de La idea de la justicia no se lleguen a establecer ciertas preferencias del autor, o sobre qué es mejor y qué peor, en aspectos tan esenciales 
como la educación, la discriminación de las mujeres o la libertad de conciencia. Aun así, y quizá a pesar suyo, Sen no está tan alejado de Rawls como insistentemente argumenta. En la Ciencia de la Lógica, Hegel afirmaba que la injusticia que con mayor frecuencia suele cometerse con el pensamiento especulativo consiste en tornarle unilateral. Sen parece tomar solamente en consideración alguna de las proposiciones de las que se compone la propuesta de Rawls, sin atender a los esfuerzos realizados por éste para enmendar y extender su teoría tanto como le fue posible en asuntos tan discutidos como "el velo de la ignorancia", la justicia entre generaciones o el derecho de gentes. Esta sospecha se fundamenta en la ausencia casi total de referencias de A. Sen al trabajo posterior a la Teoría de la Justicia de Rawls. Tampoco Martha Nussbaum encuentra acomodo en las referencias de Sen, no al menos como sus aportaciones al enfoque de las capacidades creemos que merecen. Su lista de diez capacidades centrales, publicada por primera vez en el año $2000^{18}$, suponen una propuesta normativa firmemente argumentada sobre las capacidades humanas y la centralidad que todas y cada una de las personas deben tener en las decisiones y acciones políticas. La idea de la justicia de Amartya Sen, al ser comparado con el trabajo desarrollado por Nussbaum para establecer esta enumeración de mínimos que cualquier sociedad debería cumplir para ser considerada como una sociedad decente, queda situado en el marco de un tratado teórico, pues no aporta herramientas para la realización, más allá de su brillante y riquísima exposición sobre el valor de la argumentación razonada.

La edición en castellano incluye la traducción de las notas, y de sus referencias en castellano cuando existen. Sin embargo, los completos y útiles índices tanto de nombres como de temas, mantienen la referencia a las páginas de la edición original, lo que hace que las búsquedas sean algo tediosas. La traducción es correcta aunque no muy detallista, por ejemplo en la ya mencionada distinción entre "freedom" y "liberty", o "equity" y "fairness", o en la traducción literal de "parrochialism" por "parroquialismo", o "justness" por "justeza". Todo ello hace recomendable, en caso de que se precise un análisis preciso de estos matices, tener a mano la edición original.

Aún cuando cada resumen es en última instancia un acto de barbarie, resulta muy útil y especialmente provechoso este ejercicio de supersimplificación ordenada. Espero que esta nota alcance a cumplir mínimamente la segunda parte de esta máxima de Amartya Sen y que ello anime a la lectura, si no relectura, de La idea de la justicia.

18 Nussbaum, M.C. (2000) Las mujeres y el desarrollo humano, Barcelona 2002, Herder, pp. 120-123 


\section{Referencias bibliográficas}

Nussbaum, M.C. (2000) Las mujeres y el desarrollo humano, Barcelona 2002, Herder.

RaWLS, J. (1971) Theory of Justice, Belknap, Harvard (EE. UU.).

- (1996) El liberalismo político, Barcelona, Crítica.

- (2001) El derecho de gentes, Barcelona, Paidós.

- (2002) La justicia como equidad, una reformulación, Barcelona, Paidós.

SEN, A. (2009) The Idea of Justice, Londres, Penguin.

- (2010) La idea de la justicia, traducción del inglés de Hernando VALENCIA VILA, Madrid, Taurus.

SMITH, A. (1759) La teoría de los sentimientos morales, edición a cargo de Carlos Rodríguez Braun, Madrid 1997, Alianza.

WollstoneCRAft, M. (1792) Vindicación de los derechos de la mujer, edición a cargo de Isabel BURDIEL, Madrid 2000, Cátedra. 